GorRILI, R. H. (1957). J. gen. Microbiol. 17, 254-266

\title{
Studies on Lysogeny in Staphylococci
}

\author{
BY R. H. GORRILL \\ Department of Bacteriology, Guy's Hospital Medical School, London, S.E. 1
}

\begin{abstract}
SUMMARY: A staphylococcus typing phage (NCTC 8426) was found to form lysogenic systems with three strains of staphylococci. In the case of two of these strains, staphylococci lysogenically infected with the typing phage were now lysed by the temperate phages they had previously carried. This loss of immunity was due to the replacement of the originally carried prophage by the prophage of phage 8426 . This process could be reversed. With the third strain of staphylococcus the lysogenic offspring were not lysed by their original temperate phage, which they continued to carry. The NCTC phage 8426 appeared to undergo a modification when grown on the three strains described here, following which a few particles $\left(c .1\right.$ in $\left.5 \times 10^{4}\right)$ were able to lyse cells lysogenically infected with phage 8426. These virulent phage particles were maintained by propagation on lysogenic host cells but they reverted to the normal 8426 phage when grown on the non-lysogenic indicator. Finally phage 8426 showed another modification of its host range when grown on one of the three strains, this change was reversed when the modified phage was propagated on its normal host Staphylococcus aureus NCTC 8354.
\end{abstract}

During a recent study of ultraviolet irradiation of staphylococci (Gorrill \& Gray, 1956) a number of staphylococci were lysogenically infected with typing phages. It was found that some combinations gave rise to lysogenic systems that were not completely immune to the carried phage. One such system has now been studied in more detail and the findings are recorded in this paper.

Staphylococcus-typing phage NCTC 8426 (phage 76, not now much used in typing, serological group F) is normally propagated upon Staphylococcus aureus NCTC 8354. When the phage and the sensitive staphylococcus are grown together it is possible to isolate colonies of staphylococci which appear to be lysogenically infected with phage 8426, and are quite stable on subculture. These staphylococci will be designated 8354/8426. When such staphylococci are tested for immunity to phage 8426 (grown on $S$. aureus 8354 ) they are found to show about one plaque for every $10^{5}$ plaques formed on a fully sensitive indicator, e.g. staphylococcus 8354 . When a plaque is picked from the lysogenic culture and grown in broth a phage is produced which gives about equal numbers of plaques when assayed on staphylococcus 8354 and staphylococcus 8354/8426. There exist, therefore, two forms of phage 8426. Occasionally cultures of staphylococci have been isolated which are lysogenically infected with phage 8426, but do not show this phenomenon, that is they appear to be totally resistant to both forms of phage 8426.

Further study of staphylococcus $8354 / 8426$ showed that it is sensitive to phages to which the parent staphylococcus 8354 is resistant, the most striking example of this being the sensitivity of staphylococcus 8354/8426 to the temperate phage carried by the parent staphylococcus 8354 , a phage which one would expect staphylococcus 8354/8426 itself to carry (Gorrill, 1956). 
Phage 8426 is active against a number of strains of staphylococci; one other strain was found in which infection with phage 8426 rendered the bacteria sensitive to phages they previously carried. It is believed that these observations can be explained by the replacement of the normal prophage by prophage of phage 8426 during infection leading to lysogenization.

\section{METHODS}

The original medium, buffer and counting methods were described previously by Gorrill \& Gray (1956). Unfortunately one of the constituents of the medium (Pronutrin, an enzymic digestion of casein, Herts Pharmaceuticals Ltd., Welwyn Garden City, Herts) ceased to be made during the course of these experiments. The remaining Pronutrin was reserved for broth making and a new source of agar medium sought. After prolonged trials batches of digest medium (tryptic digest of ox heart) which were completely satisfactory were obtained from the Southern Group Laboratory, Park Hospital, London, S.E. 13. Thirty $\mathrm{ml}$. of medium was used in each $10 \mathrm{~cm}$. Petri dish. The thickness of the layer of medium was found to influence the size and number of the plaques, thin plates gave very poor results even with otherwise satisfactory media. All plates were incubated at $30^{\circ}$, broth cultures at $37^{\circ}$.

Phage preparations were made by the methods described by Williams \& Rippon (1952). Phage 8426 was made on staphylococcus 8354 except where otherwise stated. Where necessary phages were purified by two or three successive single plaque isolations. All phage preparations which had to be freed of bacteria were filtered through 'Gradocol' membranes, average pore size $0 \cdot 8 \mu$ (Wright-Fleming Institute, St Mary's Hospital, London, W. 2).

Antisera were made by immunizing rabbits aged 6-9 months with high titre $\left(10^{9}-10^{10} / \mathrm{ml}\right.$.) phage stocks. The first injections were given subcutaneously, the later ones intravenously. The activity of the antisera was assayed by the methods described by Adams (1950). Phage counts were made either by spreading known dilutions over a lawn of sensitive staphylococci with a calibrated wire loop or by the soft-agar layer method.

Staphylococcus aureus strain NCTC 8354 was obtained from the National Collection of Type Cultures (NCTC), Colindale, London; strains 60, 98 and 105 from St Mary's Hospital, London, W. 2; all four strains were coagulase positive.

Typing phage 8426 was obtained from the National Collection of Type Cultures. Each of the four strains of staphylococci yielded a temperate phage; the four phages isolated will be named after the host staphylococci from which they were isolated, e.g. phage 8354 from staphylococcus $\mathbf{8 3 5 4}$. There may be other temperate phages associated with these staphylococci, but it was not possible to obtain them in pure culture. When necessary cultures were induced by irradiation with ultraviolet light. The culture was suspended in buffer and irradiated at $35 \mathrm{~cm}$. distance from a $45 \mathrm{~W}$. ultraviolet lamp (Hanovia Ltd., Slough) for $60 \mathrm{sec}$. Two $\mathrm{ml}$. samples were then added to $8 \mathrm{ml}$. broth and shaken at $37^{\circ} \mathrm{C}$. until lysis had occurred. 
Terminology. The staphylococcal typing phages and their propagating strains are identified by two different numbering systems, the laboratory numbers and the NCTC number (Williams \& Rippon, 1952). In this paper the NCTC numbers will be used in conformity with the previous paper (Gorrill \& Gray, 1956).

\section{RESULTS}

Interaction of staphylococcus 8354 with phage 8426

When phage 8426 was added to a broth culture of staphylococcus 8354 there was usually some initial clearing followed by an increase in turbidity so that after $24 \mathrm{hr}$. incubation the phage-treated culture was as dense as an untreated control. When such a culture was plated out, two different types of colony were seen, normal-looking colonies and colonies showing varying degrees of erosion and phage lysis.

The normal-looking colonies were found to be of two types, containing respectively normal non-infected staphylococci 8354 and staphylococci infected with phage 8426. Strains derived from colonies infected with phage 8426 were then examined to see if the presence of phage 8426 was due to the establishment of the lysogenic state or to phage contamination. If the culture was truly lysogenic then all the daughter colonies would show the presence of phage 8426 despite washing or treatment with antiphage serum. It was not found possible to rid these cultures of phage 8426, all daughter colonies contained the phage as was shown by replica-plating (Lederberg \& Lederberg, 1952) on to sensitive indicator staphylococci. It was concluded that phage 8426 had established a lysogenic infection in staphylococcus 8354 . In general a lysogenic strain is immune to its carried phage. When this test was carried out by spotting high-titre suspensions of phage 8426 on to the staphylococci lysogenic for phage 8426 it was found that about 1 particle in $10^{5}$ of phage 8426 would form a plaque on the lysogenic staphylococci, and so it appeared that either immunity was not complete or phage 8426 existed in a second, highly virulent, form.

A number of isolations of such lysogenic staphylococci were made and in almost all cases they behaved as described above. In the records these

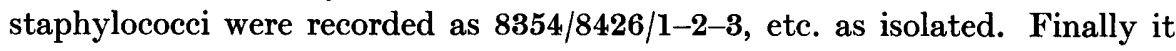
was decided that as all these cultures had the same properties it would be convenient to shorten the numbering and refer to them all as staphylococci $8354 / 6 / 1$. It will be seen later that similar types of staphylococci can be isolated from different strains infected with phage 8426 and the expression $6 / 1$ type was used to describe a staphylococcus lysogenically infected with phage 8426 but still sensitive to a small fraction ( 1 in $10^{5}$ ) of phage 8426 particles.

As staphylococci are common laboratory contaminants two steps were taken to ensure that the lysogenic forms obtained were not contaminants. First, four separate cultures of staphylococcus 8354 and phage 8426 were used and clones of the $6 / 1$ type could be regularly derived from them. Samples of such cultures were then freeze dried and at the end of the investigation a few were taken at random and sent to the Staphylococcal Reference Laboratory, 
Colindale, for phage-typing. It was found that the $6 / 1$ strains were similar to one another and could have been derived from staphylococcus 8354 .

During all the experiments with staphylococcus 8354 and phage 8426 three colonies were isolated which were lysogenically infected with phage 8426 and completely immune to high-titre suspensions of phage 8426. These were described in the records as $\mathbf{A}$ forms, they were phage typed and found to be similar to one another and different from the 6/1 strains. They will be discussed more fully later.

Table 1. The effect of cross-spotting five strains of staphylococci with their sensitivity to 8426 phage for comparison

\begin{tabular}{|c|c|c|c|c|c|}
\hline \multirow{2}{*}{ Supernatants } & \multicolumn{5}{|c|}{ Indicators } \\
\hline & 60 & 98 & 105 & 8354 & $8354 / 6 / 1$ \\
\hline 60 & 0 & CL & $\mathbf{0}$ & $\mathbf{0}$ & CL \\
\hline 98 & $\mathbf{P}_{ \pm}$ & 0 & $\mathbf{P} \pm$ & $\mathbf{P} \pm$ & $\mathbf{P} \pm$ \\
\hline 105 & $0^{-}$ & CL & $0^{-}$ & $0^{-}$ & CL' \\
\hline 8354 & SCL & SCL & SCL & $\mathbf{0}$ & $\begin{array}{l}\mathbf{P}++ \\
\text { (faint) }\end{array}$ \\
\hline $8354 / 6 / 1$ & CL & $\begin{array}{c}\text { CL } \\
\text { (weak plaques) }\end{array}$ & CL & CL & o \\
\hline 26 phage titre $10^{10}$ & $10^{10}$ & $10^{10}$ & $10^{10}$ & $10^{10}$ & $4 \times 10^{5}$ \\
\hline
\end{tabular}

\section{Change in phage sensitivity of staphylococcus $8354 / 6 / 1$}

A further interesting observation was made during a series of cross-spotting experiments, an example of which is given in Table 1. It will be seen that staphylococcus $8354 / 6 / 1$ has now become sensitive to the phages in the supernatants of staphylococci 60 and 105 (phages 60 and 105). Staphylococcus 98 has been included as it is a good indicator for the phages employed (except phage 8426 ), and it will be seen that staphylococcus $8354 / 6 / 1$ is comparable to staphylococcus 98 in its sensitivity to phages 60 and 105. Phage 8354 produces rather faint plaques on staphylococcus $8354 / 6 / 1$, but they are comparable in numbers to these produced on staphylococcus 98. This increase in phage sensitivity was found in all strains of staphylococcus 8354/6/1 tested. A further point of interest is the difference in the quality of the plaques produced on staphylococcus 98 by the supernatants of staphylococci 8354 and $8354 / 6 / 1$. The supernatant of the first gave rise to clear plaques characteristic of phage 8354 while the second gave only small ill-defined plaques similar to those given by phage 8426 . It would seem from these results that staphylococcus $8354 / 6 / 1$ had become sensitive to phages 60,105 and 8354 and lost the ability to produce phage 8354 , when compared with its parent culture staphylococcus 8354 .

From these results it was concluded that when staphylococcus 8354 was exposed to phage 8426, the staphylococci lysogenic for phage 8426 which were obtained (6/1 types) had lost prophage 8354 . This might be because 
introduction of the new prophage caused the old one to be lost; or it might be that a small proportion of the cells of strain 8354 spontaneously lost their prophage, and that only this minority of non-lysogenic cells could become lysogenic when exposed to phage 8426. A number of experiments were carried out to determine the number of cells of staphylococcus 8354 that were lysogenically infected with phage 8426 , following multiple infection. $4.5 \times 10^{7}$ cells $/ \mathrm{ml}$. of staphylococcus 8354 were exposed to $1 \cdot 2 \times 10^{9} / \mathrm{ml}$. particles of 8426 phage at $37^{\circ} \mathrm{C}$. for $30 \mathrm{~min}$. The culture was then centrifuged and the supernatant counted for 8426 phage and $4 \times 10^{8} / \mathrm{ml}$. particles remained; the multiplicity of infection therefore being about 19. The staphylococci were resuspended, diluted and aliquots spread on agar. The plates were then incubated and next day the number of colonies counted. These colonies were then replica printed against sensitive indicator media so that the number of phage-infected colonies could be scored. In this experiment $2 \times 10^{6} / \mathrm{ml}$. staphylococci survived phage treatment and $50 \%$ of these were phage infected. However, of these many were in the form of unstable or eroded colonies and in fact only $30 \%$ of the phage-infected colonies were stable and behaved on further testing as 6/1 clones. Ignoring the unstable colonies for the moment, of the $4.5 \times 10^{7} / \mathrm{ml}$. staphylococci infected, $3 \times 10^{5} / \mathrm{ml}$. formed $6 / 1$ clones, i.e. slightly less than $1 \%$. These experiments were repeated several times and gave results of this order. Hence if staphylococcus 8354 is losing prophage 8354 it should be possible to show that rather under $1 \%$ of its colonies are free of the phage. Cultures of staphylococcus 8354 were grown in broth and washed in buffer three times and then diluted and spread on digest plates and incubated to form well-developed isolated colonies. These colonies were then printed on to indicator staphylococei sensitive to phage 8354. In all, 1538 colonies were tested in this way and all contained phage 8354 and hence prophage 8354 .

It seems likely that phage 8426 plays a part in the displacement of prophage 8354 and evidence of this was obtained by the study of the eroded colonies mentioned earlier. In all experiments where staphylococcus 8354 and phage $\mathbf{8 4 2 6}$ were mixed eroded colonies were seen if the mixture was plated. In the experiment described above some $70 \%$ of the colonies infected with phage $\mathbf{8 4 2 6}$ were eroded. In a long series of experiments it was found that if a broth culture of an eroded colony was made, the culture after incubation always yielded a mixture of stable and eroded colonies. On examination the stable colonies were found to be either staphylococcus 8354 or $8354 / 6 / 1$. The rates varying slightly from experiment to experiment. In one experiment eroded colonies were passed in daily subculture for 6 months. A possible explanation of this phenomenon is as follows: If a cell containing prophage 8354 is invaded by phage 8426 it might on subdivision give rise to cells containing only prophage 8354 or prophage 8426 ; that is, it would not replicate both prophages at the same time. If such a cell had been inoculated on to a plate the colony containing both staphylococcus 8354 and $8354 / 6 / 1$ would be unstable and give rise to the appearance of eroded colonies. If grown in broth the liberation of free phage by the two lines of cells would also produce continual interaction and phage lysis. To test this theory an eroded colony was picked into broth 
and incubated at $37^{\circ} \mathrm{C}$. overnight. It was then centrifuged three times to rid it of free phage and resuspended in buffer. Dilutions were made in broth and from these dilutions plates were spread with $0 \cdot 1 \mathrm{ml}$. amounts and incubated. Suitable dilutions were examined under the microscope to ensure that the culture consisted of single, and pairs of, cocci and not clumps. The dilutions were now incubated at $37^{\circ}$ and samples taken every hour for $6 \mathrm{hr}$. and $0 \cdot 1 \mathrm{ml}$. amounts plated for colony counts. Next day it was possible to see which dilution originally contained about $100-400$ cells $/ \mathrm{ml}$. yielding 10 to 40 colonies per plate. Following the fate of the bacteria at this dilution two observations were made. First the number of colonies declined for the first $3 \mathrm{hr}$. and the number of eroded colonies also declined. After $3 \mathrm{hr}$. the colonies increased in number and none were eroded. The plates were then printed on to plates layered with staphylococcus 8354 and the colonies were found to be either lysogenic (i.e. as measured against staphylococcus 8354) or not. Colonies of both types were picked off and examined and shown to be either staphylococcus 8354 (i.e. carrying phage 8354 ) or staphylococcus $8354 / 6 / 1$. This experiment was repeated several times with similar results. It appears therefore that if cross-infection is avoided eroded colonies will finally yield a mixture of both strains of staphylococcus. As microscopy showed that the cells were in one's and two's, it seems that on incubation the cells either lyse or else divide into stable daughter cells containing only one prophage.

Phage in the supernatant of staphylococcus 8354 will form plaques on staphylococcus $8354 / 6 / 1$. The titre of phage is low and it proved impossible to isolate cells of staphylococcus $8354 / 6 / 1$ attacked by phage 8354 . If phage 8354 was propagated on any of the sensitive staphylococci available it became contaminated by the lysogenic phages of the propagating strain. However, it was found that staphylococcus 8354 was inducible and following lysis phage titres as high as $5 \times 10^{10} / \mathrm{ml}$. were obtained. Such a stock was filtered and tested for sterility and then spotted onto a lawn of staphylococcus 8354/6/1. From the bacterial growth in the centre of the spot an emulsion was prepared and freed of phage by centrifugation, and serial dilutions spread on plates. The majority of the colonies which grew were stable and all these proved to be staphylococcus $8354 / 6 / 1$. However, there were a few eroded colonies and, following the results obtained with the earlier experiments on eroded colonies, cultures were set up. From these a mixture of stable and eroded colonies were formed, and on replica plating on staphylococcus 8354 it was found that the stable colonies were of two types, lysogenic and non-lysogenic. It was then shown that the stable 'non-lysogenic' colonies were staphylococcus 8354 , i.e. they carried prophage 8354. It is therefore possible to turn staphylococcus 8354 into staphylococcus $8354 / 6 / 1$ and back by phage action. Finally, some 1200 colonies of staphylococcus $8354 / 6 / 1$ were printed against staphylococcus 8354 to ensure that the change was not due to the occurrence of a few cells that had spontaneously lost phage 8426. All the colonies tested were identical and contained 8426 phage. 


\section{Interaction of phage 8426 with staphylococci 60 and 105}

These two staphylococci were part of a series of coagulase positive staphylococci isolated from patients in hospital. As will be seen from Table 1 their behaviour is very similar and their phage type is the same. Hence it may be assumed that they are two cultures of the same organism. Until the phage typing was done it was assumed they might be different and so all the experiments described below were done with both strains.

It is possible to isolate 6/1 strains easily following the addition of phage 8426 to the culture. On one occasion a strain lysogenically infected with phage 8426 was found which was resistant to both forms of phage 8426. This is the only A strain found in this group. As with staphylococcus 8354, the 6/1 strains were now sensitive to the phage they had previously carried, and it was not possible to show the presence of the original prophage in the new strains. It was possible to isolate eroded colonies which were similar in behaviour to these already described. Briefly then, these two strains of staphylococci behave in every way like staphylococcus 8354 .

\section{Interaction of phage 8426 with staphylococcus 98}

This strain of staphylococcus was first included in these studies because it appeared to be a good indicator for 60, 105 and 8354 phage. It was known to give very small faint plaques with phage 8426 but in many ways this was an advantage as it allowed one of the first group to be counted in the presence of large amounts of phage 8426. When the action of phage 8426 on staphylococcus 98 was studied it was found that lysogenic strains could be produced and that they could be classified as 6/1 strains because they were sensitive to a few particles in stocks of phage 8426. Similarly, there was isolated one colony which failed to be lysed by the second form of phage 8426 and was classified as an ' $A$ ' strain.

There was, however, one important difference observed with the 98/6/1 strains, they were still immune to 98 phage and in fact 98 phage could be detected in their supernatant. In this case phage 8426 had not displaced the original prophage. Also while eroded colonies were seen in the early subcultures, they quickly disappeared on further subculture and yielded only 98/6/1 strains.

However staphylococcus 98 had one interesting property not shown by the other staphylococci described here, in that it seemed able to modify phage 8426 when it was propagated on it. If a lawn of staphylococcus 98 was inoculated with a dilution of phage 8426 so as to give discrete plaques it was noticed that a few were much clearer and bigger than the others. If such a plaque was picked out with a pasteur pipette into broth, shaken and used to inoculate a fresh lawn of staphylococcus 98 these plaques were all well defined. However, if the phage derived from a plaque of phage 8426 on staphylococcus 98 was counted on both staphylococcus 98 and staphylococcus 8354 it was found that it now gave a titre on staphylococcus 98 between 10 and 100 times higher than that on staphylococcus 8354. This difference was accentuated by 
several passages on staphylococcus 98 when the ratio would rise to 1000 to 1 or higher. If, however, a plaque that did appear on staphylococcus 8354 was taken it would contain phage which gave equal number of plaques on staphylococci 98 and 8354. This example of host modification of phage is less clear-cut than those seen with other phage systems but similar results have been found with other staphylococcal phages and will be discussed briefly later. Its main interest in this work was to render staphylococcus 98 an unsatisfactory strain for propagating phage $\mathbf{8 4 2 6}$ or for testing single plaques of phage $\mathbf{8 4 2 6}$.

\section{' $A$ ' strains}

There were in all five strains which were resistant to both types of 8426 phage while being lysogenically infected with phage 8426. Three were derived from staphylococcus 8354 and one each from staphylococci 105 and 98 . When phage-typed they were lysed only by typing phages 53 or 53 and 75 and so were very similar. They were tested for the presence of the original prophage and like the $6 / 1$ strains the 8354 and $105 \mathrm{~A}$ strains had lost their original prophage while the $98 \mathrm{~A}$ strain had not. When tested with phages $60,98,105$ and 8354 , all except $98 \mathrm{~A}$ were sensitive to phage 98 , none was sensitive to phage 8354 and the results with the other two were variable.

All five ' $A$ ' strains were tested with phages $8426,8426 \mathrm{v}$ (described later) and phage 8354 and were shown to adsorb these phages as readily as the 6/1 strains, so their apparent immunity was not due to failure of the phage to adsorb. In one particular they were all unusual; when the supernatant of an overnight broth culture was examined it was found to contain about $10^{7}$ phage particles/ml. which formed plaques on staphylococcus 8354 and was similar to phage 8426 in appearance. The supernatant however contained phage particles which would form plaques on the $6 / 1$ strains, there were about $10^{2}-10^{3}$ of these particles $/ \mathrm{ml}$. As will be seen later the supernatants of $6 / 1$ strains do not contain particles which form plaques on $6 / 1$ strains. It appears therefore that these $\mathbf{A}$ strains are different from the $6 / 1$ strains in their phage sensitivity and in the production of a small number of altered forms of 8426 phage.

\section{The two forms of 8426 phage}

As was described earlier if high titre suspensions of phage 8426 are made on staphylococcus 8354 and then counted on lawns of staphylococci 8354 and $8354 / 6 / 1$, it was found that a small fraction would form plaques on the $6 / 1$ strains. If single plaques on a $6 / 1$ lawn were taken they were found to contain phage which gave rather more plaques on staphylococcus $8354 / 6 / 1$ than it did on staphylococcus 8354 . This strain of phage 8426 which was capable of lysing cultures lysogenically infected with phage 8426 was designated phage $8426 \mathrm{~V}$ $(\mathrm{v}=$ virulent). Phage $8426 \mathrm{v}$ would grow equally well on all $6 / 1$ strains including those derived from staphylococci 60, 98 and 105.

Phage $8426 \mathrm{v}$ was made in bulk by propagation on lawns of staphylococcus $8354 / 6 / 1$. It was found that if plaques formed by phage $8426 \mathrm{v}$ on staphylococcus 8354 were taken they contained very little phage $8426 \mathrm{v}$ and most of the phage particles were phage 8426. It appeared therefore that the change of 
phage 8426 to phage $8426 \mathrm{v}$ and back was determined in part at least by the strain of staphylococcus used for propagation. The reversal was also seen if staphylococci 60,98 or 105 were substituted for staphylococcus 8354 .

In order to study further the part played by the host cells in this change two groups of experiments were carried out. First, using the phage isolated from single plaques lawns of staphylococci were flooded and small volumes of high titre phage produced. These stocks of phage were then counted on lawns

Table 2. Stocks of phages 8426 and $8426 v$ counted on indicators staphylococci 8354 and 8354/6/1, figures show plaque forming particles per $\mathrm{ml}$.

\begin{tabular}{|c|c|c|c|c|c|c|}
\hline \multirow{2}{*}{$\begin{array}{c}\text { Stocks of } \\
\text { phage } \\
8426\end{array}$} & \multicolumn{3}{|c|}{ Cultures of staphylococcus 8354} & \multicolumn{3}{|c|}{ Cultures of staphylococcus $8354 / 6 / 1$} \\
\hline & 1 & $\mathbf{2}$ & $\mathbf{3}$ & 1 & 2 & 3 \\
\hline 1 & $2 \times 10^{9}$ & $1 \cdot 2 \times 10^{9}$ & $2 \cdot 4 \times 10^{9}$ & $4.2 \times 10^{4}$ & $5 \cdot 1 \times 10^{4}$ & $3 \cdot 3 \times 10^{4}$ \\
\hline $\mathbf{2}$ & $2 \times 10^{9}$ & $2 \cdot 1 \times 10^{9}$ & $2.6 \times 10^{9}$ & $3.3 \times 10^{4}$ & $6 \cdot 1 \times 10^{4}$ & $8.7 \times 10^{4}$ \\
\hline $\mathbf{3}$ & $9 \times 10^{9}$ & $6 \times 10^{9}$ & $8 \times 10^{9}$ & $1.5 \times 10^{5}$ & $2 \times 10^{5}$ & $6 \times 10^{4}$ \\
\hline 4 & $2 \times 10^{9}$ & $2 \cdot 8 \times 10^{9}$ & $\mathbf{2 . 9} \times 10^{9}$ & $4 \times 10^{4}$ & $2 \cdot 2 \times 10^{4}$ & $3 \cdot 1 \times 10^{4}$ \\
\hline 5 & $3 \times 10^{10}$ & $2 \cdot 7 \times 10^{10}$ & $1.9 \times 10^{10}$ & $2.5 \times 10^{5}$ & $4.1 \times 10^{5}$ & $4.9 \times 10^{5}$ \\
\hline \multicolumn{7}{|l|}{$\begin{array}{l}\text { Stocks of } \\
\text { phage } \\
8426 \mathrm{v}\end{array}$} \\
\hline 1 & $5.5 \times 10^{7}$ & $6.0 \times 10^{7}$ & $3.3 \times 10^{7}$ & $4.1 \times 10^{7}$ & $8.9 \times 10^{7}$ & $4 \cdot 7 \times 10^{7}$ \\
\hline 2 & $1.4 \times 10^{7}$ & $1 \cdot 1 \times 10^{7}$ & $1.2 \times 10^{7}$ & $5 \cdot 1 \times 10^{7}$ & $6.8 \times 10^{7}$ & $5.7 \times 10^{7}$ \\
\hline 3 & $1 \cdot 1 \times 10^{10}$ & $1.2 \times 10^{10}$ & $9.8 \times 10^{9}$ & $2 \cdot 1 \times 10^{10}$ & $2.9 \times 10^{10}$ & $1.9 \times 10^{10}$ \\
\hline 4 & $5.9 \times 10^{7}$ & $7.5 \times 10^{7}$ & $6.5 \times 10^{7}$ & $1.1 \times 10^{8}$ & $2 \cdot 1 \times 10^{8}$ & $7 \cdot 7 \times 10^{8}$ \\
\hline 5 & $1.3 \times 10^{9}$ & $1.9 \times 10^{9}$ & $1.5 \times 10^{9}$ & $1.6 \times 10^{9}$ & $3.3 \times 10^{9}$ & $9.7 \times 10^{8}$ \\
\hline
\end{tabular}

of staphylococci 8354 and $8354 / 6 / 1$ which had been grown up from very small inocula (an overnight broth culture was diluted $10^{-6}$ in broth and then $1 \mathrm{ml}$. was added to $9 \mathrm{mls}$. for each stock and incubated). The counts were carried out on the same day with five cultures of each staphylococcus. Only a portion of the results is shown in Table 2 . It will be seen that with high titre stocks of phage 8426 about 1 in $5 \times 10^{4}$ particles form plaques on staphylococcus $8354 / 6 / 1$. With phage $8426 \mathrm{v}$ there were commonly two to four times as many plaques formed on staphylococcus $8354 / 6 / 1$ as on staphylococcus 8354 . These results were not influenced by the use of different subcultures of the two staphylococci.

In the other experiments single plaques were taken with a pasteur pipette, emulsified in buffer $(10 \mathrm{ml}$.) and the number of phage particles counted on several subcultures of the two indicator staphylococci as described above. The results are illustrated in Table 3 which shows that the change from phage $8426 \mathrm{v}$ to phage 8426 was related to the host cell (staphylococcus 8354 or $8354 / 6 / 1$ ) on which it was grown. Further experiments were done in which aged cultures and very young shaken cultures were used and in no case was a consistent trend seen which would allow one to say that the bacterial cultures varied significantly in the degree to which they brought about this change.

Finally, attempts were made to produce one-step growth curves but as is common in this group of phages (Rountree, 1955) infection of sensitive cells in broth is not always followed by a burst. However, on two occasions it was 
found that when staphylococcus 8354 was infected with a low $(0 \cdot 1)$ multiplicity of phage $8426 \mathrm{v}$ and diluted suitably a burst followed at about $90 \mathrm{~min}$. with the liberation of an average of 18 phage particles per infected cell. These particles were all phage 8426 .

\section{Table 3. Results of counting the phage content of various plaques on} different cultures of the two indicators

$A=$ plaques of 8426 phage from lawns of staphylococcus $8354 ; B=$ plaques of 8426 phage from lawns of staphylococcus $8354 / 6 / 1 ; \mathrm{C}=$ plaques of $8426 \mathrm{v}$ phage from lawns of staphylococcus 8354; $D=$ plaques of $8426 \mathrm{v}$ phage from lawns of staphylococcus 8354/6/1.

\begin{tabular}{|c|c|c|c|c|c|c|}
\hline \multirow[b]{2}{*}{ Plaque } & \multicolumn{3}{|c|}{ Cultures of staphylococcus 8354} & \multicolumn{3}{|c|}{ Cultures of staphylococcus $8354 / 6 / 1$} \\
\hline & 1 & 2 & 3 & 1 & 2 & 3 \\
\hline A 1 & $7 \times 10^{6}$ & $2.5 \times 10^{6}$ & $5 \times 10^{6}$ & $7 \times 10^{2}$ & $2 \times 10^{2}$ & $4 \times 10^{2}$ \\
\hline A2 & $2.5 \times 10^{7}$ & $1.6 \times 10^{7}$ & $2.0 \times 10^{7}$ & $1 \cdot 1 \times 10^{3}$ & $3.5 \times 10^{3}$ & $1.2 \times 10^{3}$ \\
\hline A3 & $6 \cdot 1 \times 10^{6}$ & $6 \cdot 3 \times 10^{6}$ & $4.1 \times 10^{6}$ & $3 \times 10^{2}$ & $3 \times 10^{2}$ & $4 \times 10^{2}$ \\
\hline B 1 & $7 \cdot 6 \times 10^{5}$ & $8 \cdot 1 \times 10^{5}$ & $4.9 \times 10^{5}$ & $2 \cdot 1 \times 10^{6}$ & $1.5 \times 10^{6}$ & $2 \cdot 4 \times 10^{6}$ \\
\hline B2 & $2 \cdot 2 \times 10^{5}$ & $4 \times 10^{4}$ & $3 \times 10^{5}$ & $1.9 \times 10^{5}$ & $2 \cdot 4 \times 10^{5}$ & $4.0 \times 10^{5}$ \\
\hline B3 & $4.8 \times 10^{4}$ & $2 \cdot 1 \times 10^{5}$ & $8.9 \times 10^{4}$ & $3.0 \times 10^{5}$ & $8.8 \times 10^{4}$ & $3.0 \times 10^{5}$ \\
\hline $\mathrm{C}_{1}$ & $6.0 \times 10^{6}$ & $7 \cdot 2 \times 10^{6}$ & $4.1 \times 10^{6}$ & $2 \times 10^{2}$ & $3 \times 10^{2}$ & $8 \times 10^{2}$ \\
\hline $\mathrm{C2}$ & $4.5 \times 10^{6}$ & $5 \cdot 1 \times 10^{6}$ & $8.0 \times 10^{6}$ & $9 \times 10^{2}$ & $0 *$ & $8 \times 10^{2}$ \\
\hline C3 & $1.1 \times 10^{7}$ & $8.0 \times 10^{6}$ & $2.5 \times 10^{6}$ & $7 \times 10^{2}$ & $6 \times 10^{2}$ & $2 \times 10^{2}$ \\
\hline D1 & $2.9 \times 10^{5}$ & $3.5 \times 10^{5}$ & $8.0 \times 10^{4}$ & $4.4 \times 10^{5}$ & $3.1 \times 10^{5}$ & $6.6 \times 10^{5}$ \\
\hline $\mathrm{D} 2$ & $4.4 \times 10^{5}$ & $6 \cdot 1 \times 10^{5}$ & $2.0 \times 10^{5}$ & $6.2 \times 10^{5}$ & $8.1 \times 10^{5}$ & $9 \times 10^{5}$ \\
\hline D3 & $6.7 \times 10^{5}$ & $2 \cdot 1 \times 10^{5}$ & $4 \cdot 1 \times 10^{5}$ & $1.6 \times 10^{6}$ & $8.4 \times 10^{5}$ & $2.0 \times 10^{6}$ \\
\hline
\end{tabular}

* Less than 100 phage particles $/ \mathrm{ml}$.

All attempts to isolate staphylococci lysogenically infected with phage $8426 \mathrm{v}$ were unsuccessful. It was thought that it might play some part in the formation of the ' $A$ ' strains which do liberate a small proportion of their phage as $8426 \mathrm{v}$, unlike the $6 / 1$ strains as described below.

At this point it is convenient to review the evidence that phage $8426 \mathrm{v}$ is in fact modified phage 8426. Briefly, on indicators such as staphylococci 98, 8354 and 105 their plaques are similar, they are inactivated at the same rate by antisera and are equally heat sensitive. They adsorb at the same rate on to both staphylococcus 8354 and $8354 / 6 / 1$.

Normally phages propagated on staphylococcus 8354 while contaminated with phage 8354 would appear pure on lysogenic offspring of staphylococcus 8354 as these cultures are usually immune to the temperate phages of the parent. However in this case, owing to the removal of prophage 8354 in the making of the staphylococcus $8354 / 6 / 1$ strains, phage 8354 could and in fact did multiply in staphylococcus $8354 / 6 / 1$. It was shown that phage $8426 \mathrm{v}$ was not an adapted form of phage $\mathbf{8 3 5 4}$ by the fact that they were serologically different and if plaques of phage 8354 grown on staphylococcus 8354/6/1 were taken and assayed on staphylococcus 98 , plaques of phage 8354 were seen, together with the small plaques of phage 8426 derived from the surrounding bacteria. There was no evidence therefore that phage 8354 present in phage 8426 as a contaminant in any way adapted during growth on staphylococcus $8354 / 6 / 1$ and was mistaken for a virulent form of phage 8426 . 
If the stocks of phage 8426 made on staphylococcus 8354 were examined it was seen that the virulent variant was present in all of them in the ratio of 1 in $10^{5}$ or more. Now if broth cultures of staphylococcus $8354 / 6 / 1$ were centrifuged the supernatant was found to contain about $10^{7} 8426$ phage particles $/ \mathrm{ml}$. but no $8426 \mathrm{v}$ phage. Similarly, if cultures of staphylococcus $8354 / 6 / 1$ are induced large quantities of phage 8426 are released $\left(>10^{10} / \mathrm{ml}\right.$.), but in fifteen tests no $8426 \mathrm{v}$ phage was found. The possibility that phage $8426 \mathrm{v}$ was the result of a recombination between phages 8354 and 8426 was considered. It was rejected on the grounds that strains of phage $8426 \mathrm{v}$ could be made on staphylococcus 60 and 105 and less well on staphylococcus 98 and these strains of phage $8426 \mathrm{v}$ appeared identical. On the other hand, the prophages in these staphylococci were certainly different. Finally, unlike the 6/1 strains, the $5 \mathrm{~A}$ strains did seem to produce spontaneously a small proportion of phage $8426 \mathrm{v}$.

\section{DISCUSSION}

There are two examples of host modification of phage 8426 in this paper. Staphylococcus 98 produces a variant which forms plaques to a much lower titre on staphylococcus 8354 than on staphylococcus 98 , while staphylococcus $8354 / 6 / 1$ appears to produce rare variants which are virulent.

The ability of some strains of bacteria to modify the phage propagated on them has been known for some time and was reviewed by Luria (1953). Very briefly, if a phage is grown for only one cycle in these modifying strains, it is changed and can now only rarely form plaques on its original indicator. However, these rare plaques contain normal phage and it can be shown that the return to normality follows a single cycle of growth. The ability of a few phage particles modified by the bacteria to grow on the original host is due to the presence in the original host of a few cells capable of supporting the growth of the phenotypically modified phage particles. The number of these cells varies with the physiological state of the culture and is also modified by irradiation.

In an extensive study of the typing phages of Salmonella typhi Anderson and his co-workers $(1953 a, b, 1955,1956)$ have shown that adaptation of a single phage to form a series of specific typing phages involves in some cases phenotypic modification, in others selection of host range mutants and in still others both processes. In general cultures which were lysogenically infected with unrelated ' $O$ ' phages selected host-range mutants while the nonlysogenic cells selected phenotypic variants.

In the cases reported here the results are less clear-cut. Staphylococcus 98 modifies phage $\mathbf{8 4 2 6}$ but the effect is strengthened by repeated passage. This has been seen with other staphylococcal phages (Rountree, 1956). Further study of the modifying effect of staphylococcus 98 was not made.

Considerably greater study was devoted to the change of phage 8426 to phage $8426 \mathrm{v}$. It was essential first to ensure that the appearance of phage ' $8426 \mathrm{v}$ ' was not due in fact to a contaminant phage picked up during propagation of phage 8426 . The most likely phage would have been phage 8354 but 
it is fairly certain that this possibility may be excluded. Also by complex crossing of phages from one indicator to another it seemed possible to show that the change of phage 8426 to $8426 \mathrm{v}$ was due to a modification of phage 8426 by the propagating staphylococci and not to picking up a contaminant phage. As described earlier the change occurred during growth on a sensitive propagating strain. Owing to the difficulties of producing single growth cycles it was not possible to analyse the change in detail, but it was possible in two cases to show the change of phage $8426 \mathrm{v}$ to phage 8426 in one-step growth curves. Since it is known that the number of cells capable of supporting the growth of phenotypically modified phage varies with the physiological state of the culture, it was expected that the ratio of phage 8426 to $8426 \mathrm{v}$ in different stocks would vary on different cultures of the same staphylococcus. Such was not the case, although this negative evidence would not justify one in rejecting the hypothesis of phenotypic change. On the other hand, the reversal of phage $8426 \mathrm{v}$ to 8426 when grown on staphylococcus 8354 was so constant a feature as to exclude the possibility that the change resulted from mutation. For it has been shown that if a culture selects a mutant phage then if that mutant is now propagated on the original host it retains its character. Phage $8426 \mathrm{v}$ was thought to be a virulent phage (Jacob et al. 1953) in that it never formed a lysogenic system and it lysed cells containing the prophage of its temperate variant (Jacob \& Wollman, 1953).

The behaviour of the $A$ strains in producing small quantities of virulent phage 8426 spontaneously was interesting, but it was by no means certain in what other fashion they differed from the corresponding 6/1 strains. It was therefore impossible to use these observations to suggest the mechanism by which phage 8426 was modified. It seems most likely that it is an example of phenotypic variation where the variant is virulent, but it would be unwise to be dogmatic in such a technically different field.

Bertani (1954) studied the problem of the interaction of prophage and invading phage. In order to ensure interaction he infected cells with mutants of a carried prophage and was able to show that substitution or double infection could follow. Boyd (1956) has suggested that the immunity a prophage confers on a cell with regard to another phage may vary from complete to one where the incoming phage displaces the original prophage, usually taking its place but sometimes not, resulting in a 'curing' of the phage infection. In the system studied here it is possible to displace either prophage if an excess of the other is present. It is interesting that the cells may contain both phages for a time but the situation is unstable and always results in daughter cells containing only one prophage. Stable double lysogenics did not occur, nor did 'curing' as far as could be seen. Presumably phage 8426, when reduced to prophage, occupies a similar position on the chromosome to prophages 60 , 105 and 8354 but different from that occupied by prophage 98 . 


\section{REFERENCES}

Adams, M. H. (1950). Methods of study of bacterial viruses. Meth. med. Res. $2,1$. Anderson, E. S. \& Felix, A. (1953 $a$ ). 'Degraded Vi strains' and variation in Vi phage II of Salmonella typhi. J. gen. Microbiol. 8, 408.

Anderson, E. S. \& Felix, A. $(1953 b)$. The Vi type-determining phages carried by Salmonella typhi. J. gen. Microbiol. 9, 65.

Anderson, E. S. \& Frazer, A. (1955). The influence of the factors determining Vi-type specificity in Salmonella typhi on the adaptation of Vi Phage II. J. gen. Microbiol. 13, 519.

Anderson, E. S. \& Frazer, A. (1956). The statistical distribution of phenotypically modifiable particles and host-range mutants in populations of Vi-phage II. J. gen. Microbiol. 15, 225.

Bertani, G. (1954). Studies on lysogenesis. III. Superinfection of lysogenic Shigella dysenteriae with temperate mutants of the carried phage. J. Bact. 67, 696 .

Boyd, J. S. K. (1956). Immunity of lysogenic bacteria. Nature, Lond. 178, 141.

Gorrill, R. H. (1956). Effect of superinfection on phage sensitivity in staphylococci. Nature, Lond. 178, 91.

Gorrill, R. H. \& Gray, R. A. (1956). The induction of bacteriophage in staphylococci. J. gen. Microbiol. 14, 167.

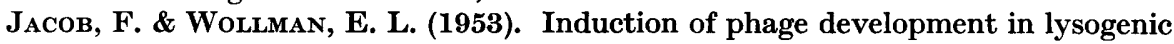
bacteria. Cold Spr. Harb. Symp. quant. Biol. 18, 101.

Jacob, F., Lwoff, A., Siminovitch, A. \& Wollman, E. L. (1953). Définition de quelques termes relatifs à la lysogénie. Ann. Inst. Pasteur, 84, 222.

Lederberg, J. \& Lederberg, E. M. (1952). Replica plating and indirect selection of bacterial mutants. J. Bact. 63, 399.

Luria, S. E. (1953). Host-induced modification of viruses. Cold Spr. Harb. Symp. quant. Biol. 18, 237.

Rountree, B. M. (1955). The role of divalent cations in the multiplications of staphylococcal bacteriophages. J. gen. Microbiol. 12, 275.

Rountree, P. M. (1956). Variations in a related series of staphylococcal bacteriophages. J. gen. Microbiol. 15, 266.

Williams, R. E. O. \& Rippon, J. E. (1952). Bacteriophage typing of Staphylococcus aureus. J. Hyg., Camb. 50, 320. 